\title{
Generation of $N$-Acyliminium ions via Intramolecular Conjugate Addition Reactions: A Strategy for the Total Synthesis of Nakadomarin A.
}

\section{Supporting Information}

\author{
Mark G. Nilson and Raymond L. Funk* \\ Department of Chemistry, Pennsylvania State University, \\ University Park, PA 16802 \\ rlf@chem.psu.edu
}

\section{General Procedures}

Unless otherwise noted, all reactions were carried out under a nitrogen atmosphere using flame-dried glassware. All moisture sensitive reagents were added via a dry syringe or cannula where possible. Anhydrous acetonitrile $\left(\mathrm{CH}_{3} \mathrm{CN}\right)$, tetrahydrofuran (THF), dichloromethane $\left(\mathrm{CH}_{2} \mathrm{Cl}_{2}\right)$, diethyl ether $\left(\mathrm{Et}_{2} \mathrm{O}\right)$, and triethylamine $\left(\mathrm{Et}_{3} \mathrm{~N}\right)$ were obtained from a solvent dispensing system. All other solvents and reagents were used as obtained from commercial sources without further purification. ${ }^{1} \mathrm{H}$ and ${ }^{13} \mathrm{C}$ NMR spectra were obtained on Bruker 300 or $400 \mathrm{MHz}$ spectrometers. Infrared spectra were obtained using a Perkin-Elmer 1600 FTIR. Chromatographic purification was performed using Sorbent Technologies silica gel 60 (230-400 mesh). Melting points were obtained on a Thomas Hoover melting point apparatus.

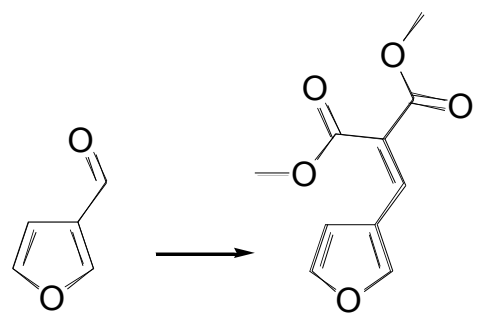

17

Diester 17. To a solution of 3-furaldehyde $(6.96 \mathrm{~mL}, 83.3 \mathrm{mmol})$ in $\mathrm{MeCN}(83 \mathrm{~mL})$ at $40{ }^{\circ} \mathrm{C}$ was added dimethylmalonate $(10.0 \mathrm{~mL}, 87.5 \mathrm{mmol})$ and ytterbium trifluoromethanesulfonate hydrate $(1.29 \mathrm{~g}, 2.08 \mathrm{mmol})$. The solution was stirred for $48 \mathrm{~h}$ then quenched with sat. $\mathrm{NaHCO}_{3}(\mathrm{aq})$ and extracted with ether. The combined organic layers were washed with brine, dried over $\mathrm{Na}_{2} \mathrm{SO}_{4}$ and concentrated to give diester $\mathbf{1 7}$ as a yellow oil $(15.8 \mathrm{~g}, 90 \%) .{ }^{1} \mathrm{H}$ NMR $\left(\mathrm{CDCl}_{3}, 300 \mathrm{MHz}\right) \delta 3.83(\mathrm{~s}, 3 \mathrm{H}), 3.90(\mathrm{~s}, 3 \mathrm{H}), 6.46$ (s, 1H), $7.44(\mathrm{~s}, 1 \mathrm{H}), 7.63(\mathrm{~s}, 1 \mathrm{H}), 7.75(\mathrm{~s}, 1 \mathrm{H}) ;{ }^{13} \mathrm{C} \mathrm{NMR}\left(\mathrm{CDCl}_{3}, 75 \mathrm{MHz}\right) \delta$ 52.5, 52.6, 108.6, 120.0, 123.5, 133.3, 144.5, 146.8, 164.5, 166.9; IR (neat) 3135, 3003, 2954, 1732, $1634,1509,1437 \mathrm{~cm}^{-1}$; HRMS $\left(\mathrm{M}^{+}\right)$calcd for $\mathrm{C}_{10} \mathrm{H}_{10} \mathrm{O}_{5} 210.0528$, found 210.0525 . 


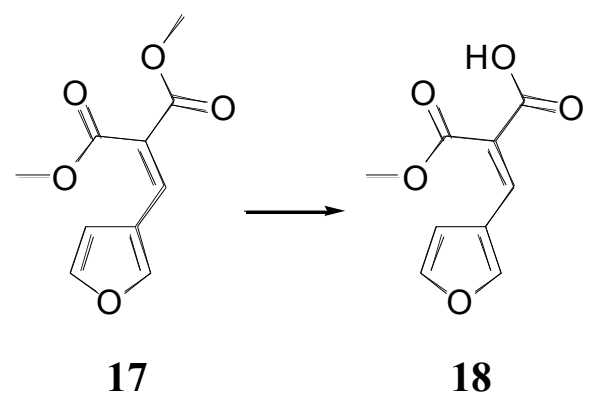

Acid 18. To a solution of diester $17(5.00 \mathrm{~g}, 23.8 \mathrm{mmol})$ in THF $(119 \mathrm{~mL})$ at $\mathrm{rt}$ was added $1 \mathrm{M} \mathrm{NaOH}$ (aq) $(96 \mathrm{~mL}, 96 \mathrm{mmol})$. The biphasic solution was stirred vigorously for $6 \mathrm{~h}$, cooled to $0{ }^{\circ} \mathrm{C}$, acidified by dropwise addition of $2 \mathrm{M} \mathrm{HCl}$ (aq) and extracted with EtOAc. The combined organic layers were dried over $\mathrm{Na}_{2} \mathrm{SO}_{4}$ and concentrated. The crude acid was recrystallized from ether to give acid $\mathbf{1 8}$ as colorless crystals (mp $\left.121-123^{\circ} \mathrm{C}, 4.18 \mathrm{~g}, 89 \%\right) .{ }^{1} \mathrm{H}$ NMR $\left(\mathrm{CDCl}_{3}, 400 \mathrm{MHz}\right) \delta 3.93$ (s, 3H), $6.55(\mathrm{~s}, 1 \mathrm{H}), 7.47$ $(\mathrm{s}, 1 \mathrm{H}), 7.84(\mathrm{~s}, 1 \mathrm{H}), 7.86(\mathrm{~s}, 1 \mathrm{H}) ;{ }^{13} \mathrm{C} \mathrm{NMR}\left(\mathrm{CDCl}_{3}, 75 \mathrm{MHz}\right) \delta 52.7,109.0,120.2$, 121.7, 136.7, 144.7, 148.2, 166.9, 169.0; IR (neat) 3133 (br), 2956, 1732, 1697, 1633, $1440 \mathrm{~cm}^{-1}$; HRMS $\left(\mathrm{MH}^{+}\right)$calcd for $\mathrm{C}_{9} \mathrm{H}_{9} \mathrm{O}_{5}$ 197.0450, found 197.0452.



Mixed anhydride 19. To a solution of acid $\mathbf{1 8}(1.62 \mathrm{~g}, 8.26 \mathrm{mmol})$ in THF $(83 \mathrm{~mL})$ at $\mathrm{rt}$ was added 4-methylmorpholine $(1.00 \mathrm{~mL}, 9.09 \mathrm{mmol})$ and isobutylchloroformate $(1.18$ $\mathrm{mL}, 9.09 \mathrm{mmol}$ ). The solution was stirred for $3 \mathrm{~h}$, quenched with sat. $\mathrm{NH}_{4} \mathrm{Cl}(\mathrm{aq})$ and extracted with $\mathrm{Et}_{2} \mathrm{O}$. The combined organic layers were washed with sat. $\mathrm{NaHCO}_{3}(\mathrm{aq})$, dried over $\mathrm{Na}_{2} \mathrm{SO}_{4}$ and concentrated to give mixed anyhydride 19 as a brown oil $(2.42 \mathrm{~g}$, 99\%). ${ }^{1} \mathrm{H}$ NMR $\left(\mathrm{CDCl}_{3}, 400 \mathrm{MHz}\right) \delta 0.97(\mathrm{~d}, J=6.7 \mathrm{~Hz}, 6 \mathrm{H}), 2.05$ (septet, $J=6.7,1 \mathrm{H}$ ), $3.91(\mathrm{~s}, 1 \mathrm{H}), 4.06(\mathrm{~d}, \mathrm{~J}=6.7 \mathrm{~Hz}, 2 \mathrm{H}), 6.52(\mathrm{~s}, 1 \mathrm{H}), 7.46(\mathrm{~s}, 1 \mathrm{H}), 7.74(\mathrm{~s}, 1 \mathrm{H}), 7.85(\mathrm{~s}$, $1 \mathrm{H}) ;{ }^{13} \mathrm{C}$ NMR $\left(\mathrm{CDCl}_{3}, 75 \mathrm{MHz}\right) \delta 18.6,27.5,52.8,75.8,108.8,120.0,121.2,137.3$, 144.9, 148.4, 148.6, 158.7, 165.4; IR (neat) 3138, 2964, 2877, 1805, 1731, 1628, 1510, 1470, 1438, $1372 \mathrm{~cm}^{-1}$; HRMS $\left(\mathrm{M}+\mathrm{Na}^{+}\right)$Calcd for $\mathrm{C}_{14} \mathrm{H}_{16} \mathrm{O}_{7} \mathrm{Na} 319.0794$, found 319.0800 . 


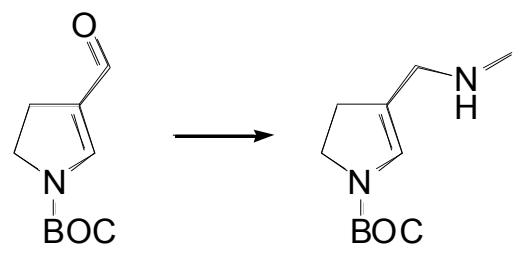

20

Amine 20. To a solution of 4-formyl-2,3-dihydropyrrole-1-carboxylic acid tert-butyl $\operatorname{ester}^{1}(5.20 \mathrm{~g}, 28.3 \mathrm{mmol})$ in $\mathrm{MeOH}(282 \mathrm{~mL})$ at $\mathrm{rt}$ was added methylamine hydrochloride $(7.63 \mathrm{~g}, 113 \mathrm{mmol})$ and triethylamine $(4.33 \mathrm{~mL}, 31.2 \mathrm{mmol})$. The solution was stirred for $3 \mathrm{~h}$ at $\mathrm{rt}$. The solution was then cooled to $0{ }^{\circ} \mathrm{C}$ and sodium borohydride $(1.18 \mathrm{~g}, 31.1 \mathrm{mmol})$ was added. The solution was stirred for $15 \mathrm{~min}$, quenched with sat. $\mathrm{NaHCO}_{3}(\mathrm{aq})$ and extracted with ether. The organic layer was dried over $\mathrm{Na}_{2} \mathrm{SO}_{4}$ and concentrated. The crude material was purified by silica gel column chromatography (Et $\left.{ }_{3} \mathrm{~N}-\mathrm{EtOAc}, 5: 95\right)$ to give amine 20 as a yellow oil $(4.87 \mathrm{~g}, 81 \%) .{ }^{1} \mathrm{H}$ NMR $\left(\mathrm{CDCl}_{3}, 400 \mathrm{MHz}\right.$, amide rotamers) $\delta 1.43$ (s, 9H), 2.39 (br s, 3H), 2.55 (br t, $J=$ $8.9 \mathrm{~Hz}, 2 \mathrm{H}), 3.21$ (br s, 2H), 3.33 (br m, 1H), 3.71 (br t, 2H), 6.32 and 6.46 (br s, $1 \mathrm{H}$, rotamers); ${ }^{13} \mathrm{C}$ NMR (CDCl3, $75 \mathrm{MHz}$, rotamers) $\delta$ 28.4, 29.9 (major) and 30.8 (minor), 35.1 (br), 45.1 (major) and 45.7 (minor), 49.3 (br), 78.9 (minor) and 79.9 (major), 120.1 (br), 126.0 (major) and 126.4 (minor), 151.4 (major) and 152.0 (minor); IR (neat) 3323 (br), 2974, 2931, 2788, 1689, 1478, $1416 \mathrm{~cm}^{-1}$; HRMS $\left(\mathrm{MH}^{+}\right)$calcd for $\mathrm{C}_{11} \mathrm{H}_{21} \mathrm{~N}_{2} \mathrm{O}_{2}$ 213.1603, found 213.1587.

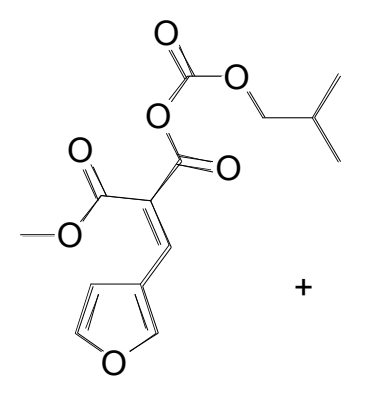

19

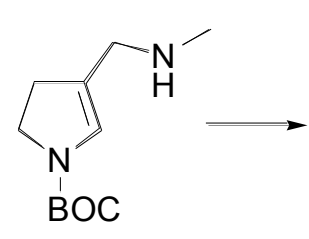

20

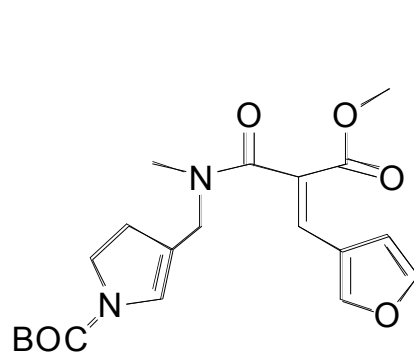

21

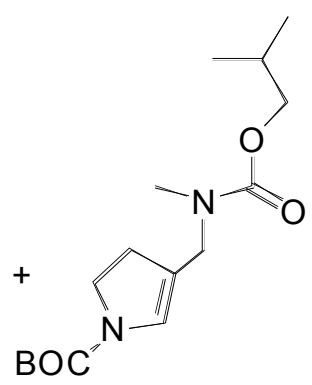

S1

Amide 21. To a solution of mixed anyhydride 19 (500 mg, $1.69 \mathrm{mmol})$ in $\mathrm{CH}_{2} \mathrm{Cl}_{2}$ (17 $\mathrm{mL})$ at $0^{\circ} \mathrm{C}$ was added amine $20(395 \mathrm{mg}, 1.86 \mathrm{mmol})$ in $\mathrm{CH}_{2} \mathrm{Cl}_{2}(1.86 \mathrm{~mL})$. The resultant solution was stirred for $2 \mathrm{~h}$ at $0{ }^{\circ} \mathrm{C}$ then quenched with sat. $\mathrm{NH}_{4} \mathrm{Cl}$ (aq). The organic layer was dried over $\mathrm{Na}_{2} \mathrm{SO}_{4}$ and concentrated. The crude material was purified by silica gel column chromatography $\left(\mathrm{Et}_{2} \mathrm{O}-\mathrm{CH}_{2} \mathrm{Cl}_{2}, 15: 85\right)$ to give amide $\mathbf{2 1}$ as a white foam $(334 \mathrm{mg}, 50 \%)$ byproduct carbamate $\mathbf{S 1}(75 \mathrm{mg}, 15 \%)$ and recovered $\mathbf{1 9}(70 \mathrm{~g}$, $14 \%)$. ${ }^{1} \mathrm{H}$ NMR $\left(d_{6}\right.$-acetone, $400 \mathrm{MHz}$, amide rotamers) $\delta 1.44$ (s, 9H), 2.47-2.64 (br app t, $2 \mathrm{H}), 2.91(\mathrm{~s}, 3 \mathrm{H}), 3.64-3.77$ (br t, $J=8.8 \mathrm{~Hz}, 2 \mathrm{H}), 3.79$ (s, 3H), 6.42-6.56 (br s, $1 \mathrm{H}$, rotamers), $6.93(\mathrm{br} \mathrm{s}, 2 \mathrm{H}), 7.60(\mathrm{t}, J=1.5 \mathrm{~Hz}, 1 \mathrm{H}), 8.17$ (br s, $1 \mathrm{H}) ;{ }^{13} \mathrm{C} \mathrm{NMR}\left(\mathrm{CDCl}_{3}, 75\right.$ $\mathrm{MHz}$, major rotamer) $\delta 28.8,30.0,36.0,45.8,49.7,52.5,80.7,112.1,117.2,120.7$, 126.4, 127.8, 133.6, 143.7, 148.1, 151.7, 164.9, 168.6; IR (neat) 3120, 2975, 2931, 1697, 
1637, 1479, 1420, 1401, $1365 \mathrm{~cm}^{-1}$; HRMS $\left(\mathrm{MH}^{+}\right)$calcd for $\mathrm{C}_{20} \mathrm{H}_{27} \mathrm{~N}_{2} \mathrm{O}_{6} 391.1869$, found 391.1893.

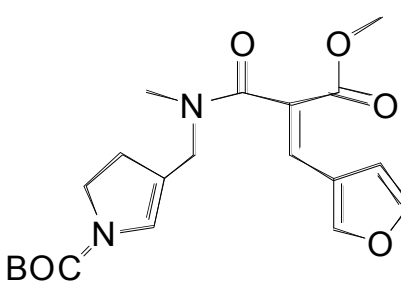

21

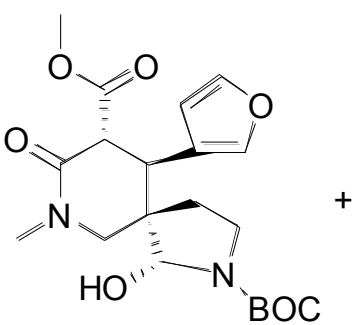

$22 \alpha$

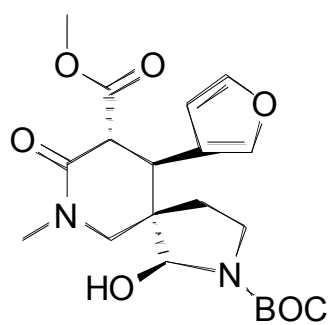

$22 \beta$

Hemi-aminals $22 \boldsymbol{\alpha}$ and 22 $\boldsymbol{\beta}$. To a solution of amide 21 (182 $\mathrm{mg}, 0.466 \mathrm{mmol}$ ) in $\mathrm{CH}_{2} \mathrm{Cl}_{2}$ $(9 \mathrm{~mL})$ at $0{ }^{\circ} \mathrm{C}$ was added scandium trifluoromethanesulfonate $(23 \mathrm{mg}, 0.046 \mathrm{mmol})$. The solution was stirred at $0{ }^{\circ} \mathrm{C}$ for $3 \mathrm{~h}$ and quenched with sat. $\mathrm{NH}_{4} \mathrm{Cl}$ (aq). The organic layer was dried over $\mathrm{Na}_{2} \mathrm{SO}_{4}$ and concentrated. The crude material was purified by silica gel column chromatography $\left(\mathrm{MeOH}_{-} \mathrm{Et}_{2} \mathrm{O}, 3: 97\right)$ to afford epimeric hemi-aminals $22 \boldsymbol{\alpha}$

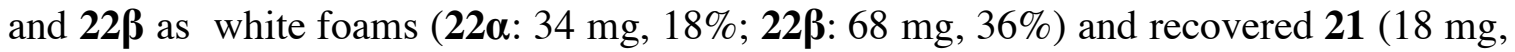
$10 \%)$. Hemi-aminal $\boldsymbol{\beta}$ was recrystallized from EtOAc/Et ${ }_{2} \mathrm{O}, \mathrm{mp} 168-170{ }^{\circ} \mathrm{C}$.

Hemi-aminal 22 $\alpha$ : HRMS $\left(\mathrm{MH}^{+}\right)$calcd for $\mathrm{C}_{20} \mathrm{H}_{29} \mathrm{~N}_{2} \mathrm{O}_{7} 409.1975$, found 409.1980.

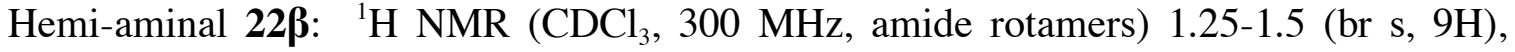
1.85-1.98 (br s, 1H), 2.13 (ddd, $J=13.0,8.60,4.33 \mathrm{~Hz}, 1 \mathrm{H}), 2.89-3.01$ (br s, 3H), 3.053.19 (br app t, 1H), 3.21-3.42 (br m, 3H), 3.45-3.56 (br s, 3H), 3.58-3.70 (br m, 1H), 3.72-3.82 (d, $J=11.4 \mathrm{~Hz}, 2 \mathrm{H}), 4.85-4.98$ (br s, $1 \mathrm{H}), 5.15-5.33$ (br s, $1 \mathrm{H}), 6.52$ (br s, $1 \mathrm{H})$, 7.47 (br s, $1 \mathrm{H}), 7.49$ (br s, $1 \mathrm{H}) ;{ }^{13} \mathrm{C} \mathrm{NMR}\left(\mathrm{CDCl}_{3}, 75 \mathrm{MHz}\right) \delta 26.3,28.3,35.4,38.5,43.6$, $46.9,52.5,52.8,53.0,80.6,84.7,110.2,120.7,140.6,143.3,155.0,164.8,169.9$; IR (neat) 3368 (br), 3130, 2976, 1742, 1687, 1644, 1501, 1478, 1432, $1392 \mathrm{~cm}^{-1}$; HRMS $\left(\mathrm{MH}^{+}\right)$calcd for $\mathrm{C}_{20} \mathrm{H}_{29} \mathrm{~N}_{2} \mathrm{O}_{7} 409.1975$, found 409.1943.

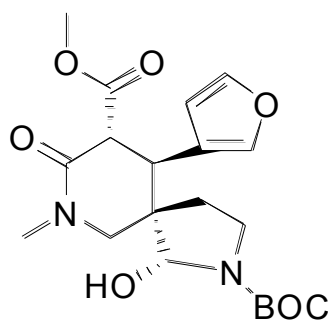

$22 \alpha$

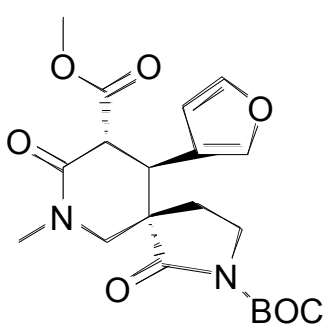

23

Imide 23. To a solution of hemi-aminal $22 \alpha(12 \mathrm{mg}, 0.029 \mathrm{mmol})$ in $\mathrm{CH}_{2} \mathrm{Cl}_{2}(1 \mathrm{~mL})$ at $\mathrm{rt}$ was added pyridinium chlorochromate $(9.5 \mathrm{mg}, 0.441 \mathrm{mmol})$. The solution was allowed to stir overnight at rt. The reaction mixture was then filtered through silica gel, washed 
thoroughly with EtOAc, and concentrated. The crude material was purified by silica gel column chromatography $\left(\mathrm{MeOH}-\mathrm{Et}_{2} \mathrm{O}, 3: 97\right)$ to give imide $\mathbf{2 3}$ as a white solid $(7 \mathrm{mg}$, 59\%). ${ }^{1} \mathrm{H} \mathrm{NMR}\left(\mathrm{CDCl}_{3}, 400 \mathrm{MHz}\right) \delta 1.49(\mathrm{~s}, 9 \mathrm{H}), 1.90$ (ddd, $\left.J=13.3,8.8,4.7 \mathrm{~Hz}, 1 \mathrm{H}\right)$, 2.08 (ddd, $J=13.3,9.0,6.6 \mathrm{~Hz}, 1 \mathrm{H}), 3.00(\mathrm{~s}, 3 \mathrm{H}), 3.12(\mathrm{~d}, J=12.4 \mathrm{~Hz}, 1 \mathrm{H}), 3.29$ (ddd, $J=11.0,9.3,4.8 \mathrm{~Hz}, 1 \mathrm{H}), 3.55(\mathrm{ddd}, J=11.0,8.8,6.6 \mathrm{~Hz}, 1 \mathrm{H}), 3.61(\mathrm{~d}, J=12.8 \mathrm{~Hz}$, $1 \mathrm{H}), 3.67(\mathrm{~s}, 3 \mathrm{H}), 3.86(\mathrm{~d}, J=12.4 \mathrm{~Hz}, 1 \mathrm{H}), 3.96(\mathrm{~d}, J=12.8 \mathrm{~Hz}, 1 \mathrm{H}), 6.33(\mathrm{~s}, 1 \mathrm{H}), 7.34$ (s, 1H), $7.36(\mathrm{~s}, 1 \mathrm{H}) ;{ }^{13} \mathrm{C}$ NMR $\left(\mathrm{CDCl}_{3}, 75 \mathrm{MHz}\right) \delta 22.7,27.9,35.2,38.5,43.1,50.3$, 51.0, 52.7, 56.1, 83.5, 110.0, 120.6, 140.8, 143.4, 149.4, 164.4, 169.3, 173.3; IR (neat) 3130, 2980, 2933, 1778, 1744, 1651, 1503, 1455, 1435, 1370, $1298 \mathrm{~cm}^{-1}$; HRMS $\left(\mathrm{MH}^{+}\right)$ calcd for $\mathrm{C}_{20} \mathrm{H}_{27} \mathrm{~N}_{2} \mathrm{O}_{7} 407.1818$, found 407.1803 .

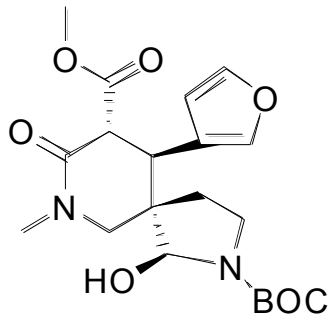

$22 \beta$

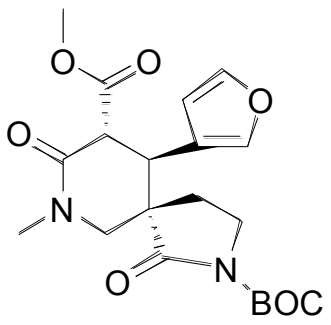

23

Imide 23. To a solution of hemi-aminal $\mathbf{2 2 \beta}(12 \mathrm{mg}, 0.029 \mathrm{mmol})$ in $\mathrm{CH}_{2} \mathrm{Cl}_{2}(1 \mathrm{~mL})$ at $\mathrm{rt}$ was added pyridinium chlorochromate $(9.5 \mathrm{mg}, 0.441 \mathrm{mmol})$. The solution was allowed to stir overnight at $\mathrm{rt}$. The reaction mixture was then filtered through silica gel, washed thoroughly with EtOAc, and concentrated. The crude material was purified by silica gel column chromatography ( $\left.\mathrm{MeOH}-\mathrm{Et}_{2} \mathrm{O}, 3: 97\right)$ to give imide $\mathbf{2 3}$ as a white solid (5 mg, $42 \%)$.

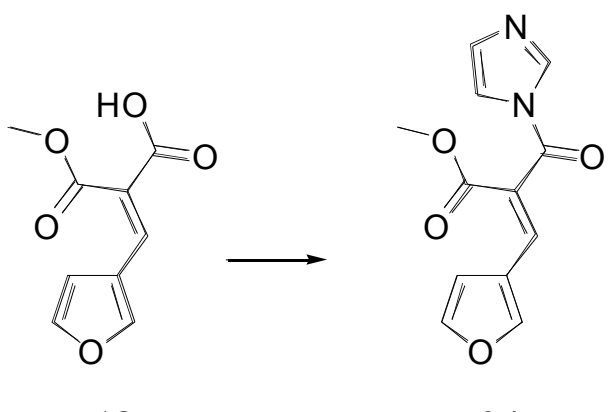

18

24

Imidazolide 24. To a solution of acid $\mathbf{1 8}(1.00 \mathrm{~g}, 5.10 \mathrm{mmol})$ in THF (20.4 mL) at rt was added 1,1'-carbonyldiimidazole $(992 \mathrm{mg}, 6.12 \mathrm{mmol})$. The solution was stirred for 15 min, quenched with $1 \mathrm{~N} \mathrm{H}_{2} \mathrm{SO}_{4}$ (aq) and extracted with ether. The combined organic layers were washed with $\mathrm{NaHCO}_{3}(\mathrm{aq})$, dried over $\mathrm{Na}_{2} \mathrm{SO}_{4}$ and concentrated to give imidazolide 24 as a tan solid $(1.09 \mathrm{~g}, 86 \%)$. ${ }^{1} \mathrm{H}$ NMR $\left(\mathrm{CDCl}_{3}, 400 \mathrm{MHz}\right) \delta 3.80(\mathrm{~s}, 3 \mathrm{H})$, $6.18(\mathrm{~s}, 1 \mathrm{H}), 7.12(\mathrm{~s}, 1 \mathrm{H}), 7.35(\mathrm{~s}, 1 \mathrm{H}), 7.50(\mathrm{~s}, 1 \mathrm{H}), 7.77(\mathrm{~s}, 1 \mathrm{H}), 7.92(\mathrm{~s}, 1 \mathrm{H}), 8.01(\mathrm{~s}$, $1 \mathrm{H}) ;{ }^{13} \mathrm{C} \mathrm{NMR}\left(\mathrm{CDCl}_{3}, 75 \mathrm{MHz}\right) \delta$ 53.0, 107.9, 116.1, 119.5, 122.5, 131.8, 136.1, 145.4, 
148.0, 163.4, 163.7; IR (neat) 3128, 2954, 1727, 1630, 1471, $1436 \mathrm{~cm}^{-1}$; HRMS $\left(\mathrm{MH}^{+}\right)$ calcd for $\mathrm{C}_{12} \mathrm{H}_{11} \mathrm{~N}_{2} \mathrm{O}_{4} 247.0719$, found 247.0736.

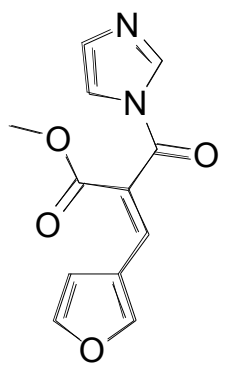

24

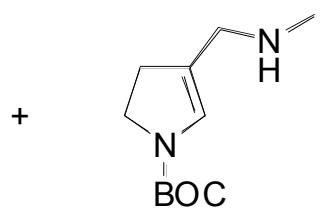

20

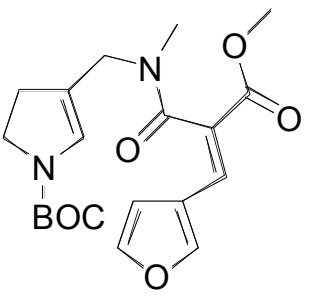

25

Amide 25. To a solution of imidazolide $24(600 \mathrm{mg}, 2.44 \mathrm{mmol})$ in $\mathrm{CH}_{2} \mathrm{Cl}_{2}(25 \mathrm{~mL})$ at $\mathrm{rt}$ was added amine $20(544 \mathrm{mg}, 2.56 \mathrm{mmol})$ in $\mathrm{CH}_{2} \mathrm{Cl}_{2}(2.5 \mathrm{~mL})$. The resulting solution was heated to $40{ }^{\circ} \mathrm{C}$ with stirring for $72 \mathrm{~h}$. The reaction was quenched with $1 \mathrm{~N} \mathrm{H}_{2} \mathrm{SO}_{4}$ (aq). The organic phase was dried over $\mathrm{Na}_{2} \mathrm{SO}_{4}$ and concentrated. Purification by silica gel column chromatography $\left(\mathrm{Et}_{2} \mathrm{O}-\mathrm{CH}_{2} \mathrm{Cl}_{2}, 15: 85\right)$ to afford amide 25 as a white foam (715 mg, 75\%). ${ }^{1} \mathrm{H}$ NMR $\left(\mathrm{CDCl}_{3}, 400 \mathrm{MHz}\right.$, amide rotamers) $\delta 1.45$ and $1.46(\mathrm{~s}, 9 \mathrm{H})$, 2.30 and 2.53-2.71 (br t, $J=9.2 \mathrm{~Hz}, 2 \mathrm{H}$ ), 2.81, 2.82, 3.01 and 3.04 (br s, 3H), 3.57-3.97 (br m, 4H), 3.79 (s, 3H), 6.29 and 6.58 (br s, 1H), 6.46 and 6.51 (br s, 1H), 7.40 (br s, $1 \mathrm{H}), 7.56$ and 7.58 (br s, $1 \mathrm{H}), 7.70$ and 7.72 (br s, $1 \mathrm{H}) ;{ }^{13} \mathrm{C} \mathrm{NMR}\left(\mathrm{CDCl}_{3}, 75 \mathrm{MHz}\right.$, major rotamer) $\delta 28.3,30.2,34.7,44.4,48.7,52.4,80.3,108.9,116.5,120.3,126.0,127.9$, 131.2, 131.6, 144.6, 146.1, 164.9, 167.1; IR (neat) 3124, 2975, 2931, 1698, 1636, 1478, 1420, $1366 \mathrm{~cm}^{-1}$; HRMS $\left(\mathrm{MH}^{+}\right)$calcd for $\mathrm{C}_{20} \mathrm{H}_{27} \mathrm{~N}_{2} \mathrm{O}_{6} 391.1869$, found 391.1847.



25

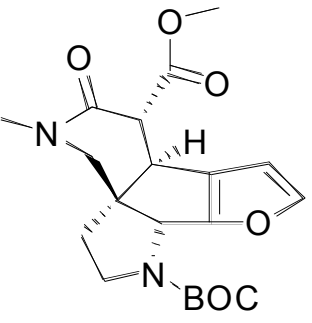

26

Tetracycle 26. To a solution of amide $25(100 \mathrm{mg}, 0.256 \mathrm{mmol})$ in $\mathrm{CH}_{2} \mathrm{Cl}_{2}(5 \mathrm{~mL})$ at 0 ${ }^{\circ} \mathrm{C}$ was added scandium trifluoromethanesulfonate $(12.6 \mathrm{mg}, 0.0256 \mathrm{mmol})$. The solution was stirred at $0{ }^{\circ} \mathrm{C}$ for $1.5 \mathrm{~h}$, allowed to warm to rt over 30 minutes and quenched with sat. $\mathrm{NH}_{4} \mathrm{Cl}(\mathrm{aq})$. The organic layer was dried over $\mathrm{Na}_{2} \mathrm{SO}_{4}$ and concentrated. The crude material was purified by silica gel column chromatography ( $\mathrm{MeOH}-\mathrm{Et}_{2} \mathrm{O}, 3: 97$ ) and recrystallized from EtOAc to afford tetracycle 26 as colorless crystals (mp 180-182 $\left.{ }^{\circ} \mathrm{C}, 80 \mathrm{mg}, 80 \%\right) .{ }^{1} \mathrm{H} \mathrm{NMR}\left(\mathrm{CDCl}_{3}, 300 \mathrm{MHz}\right) \delta 1.50$ (br s, 9H) 1.89 (ddd, $J=12.8,7.5$, $7.5 \mathrm{~Hz} 1 \mathrm{H}$ ), 2.08 (ddd, $J=12.8,6.1,6.1 \mathrm{~Hz}, 1 \mathrm{H}), 3.02$ (s, 3H), 3.26-3.55 (m, 4H), 3.56$3.76(\mathrm{~m}, 2 \mathrm{H}), 3.81(\mathrm{~s}, 3 \mathrm{H}), 4.63$ and 4.77 (br s, $1 \mathrm{H}$, rotamers), $6.10(\mathrm{~d}, J=1.91 \mathrm{~Hz}, 1 \mathrm{H})$, $7.53(\mathrm{~s}, 1 \mathrm{H}) ;{ }^{13} \mathrm{C} \mathrm{NMR}\left(\mathrm{CDCl}_{3}, 75 \mathrm{MHz}\right) \delta 28.3,35.6,36.5,43.8,44.3,52.7,54.1,55.7$, $61.7,62.7,80.2,106.7,127.6,148.2,154.0,155.6,167.3,169.5$; IR (neat) 2975, 1742, 
1670, 1487, 1436, 1399, $1365 \mathrm{~cm}^{-1}$; HRMS $\left(\mathrm{MH}^{+}\right)$calcd for $\mathrm{C}_{20} \mathrm{H}_{27} \mathrm{~N}_{2} \mathrm{O}_{6} 391.1869$, found 391.1850 .

$\underline{\text { References }}$

1. (a) Greshock, T. G.; Funk, R. L. J. Am. Chem. Soc. 2006, 128, 4946. For another preparation, see: (b) Magnus, P.; Fielding, M. R.; Wells, C.; Lynch, V. Tet. Lett. 2002, 43, 947. 